Typeset with jpsj2.cls $<$ ver.1.2 $>$

Full PAPER

\title{
Griffiths Inequalities for Ising Spin Glasses on the Nishimori Line
}

\author{
Hidetsugu KitATANi \\ Department of General Education, Nagaoka University of Technology, Nagaoka, Niigata 940-2188
}

\begin{abstract}
The Griffiths inequalities for Ising spin glasses are proved on the Nishimori line with various bond randomness which includes Gaussian and $\pm J$ bond randomness. The proof for Ising systems with Gaussian bond randomness has already been carried out by Morita et al, which uses not only the gauge theory but also the properties of the Gaussian distribution, so that it cannot be directly applied to the systems with other bond randomness. The present proof essentially uses only the gauge theory, so that it does not depend on the detail properties of the probability distribution of random interactions. Thus, the results obtained from the inequalities for Ising systems with Gaussian bond randomness do also hold for those with various bond randomness, especially with $\pm J$ bond randomness.
\end{abstract}

KEYWORDS: spin glass, Griffiths inequality, Ising model, Nishimori line, gauge theory, thermodynamic limit

\section{Introduction}

The Griffiths inequalities make significant contributions to the understanding of phase transition for ferromagnetic Ising models. ${ }^{1)}$ For one formulation, the Griffiths inequalities are written as

$$
\frac{d P}{d J_{B}} \geq 0
$$

and

$$
\frac{d\left\langle S_{C}\right\rangle}{d J_{B}} \geq 0
$$

where $J_{B}$ is a positive interaction and $S_{C}=\prod_{i \in C} S_{i}\left(S_{i}= \pm 1\right)$ is a product of Ising variables for arbitrary subset of the sites of the system. The above inequalities state that the pressure, $P(=\log Z)$, and the correlation function, $\left\langle S_{C}\right\rangle$, are monotonic increasing functions of the strength of any interaction, $J_{B}$. Using the two inequalities, various results can be proved, for example, the existence of the thermodynamic limit of the pressure per unit volume and correlation functions. They also give significant insights for the existence of ferromagnetic phase transition.

For random Ising systems which have both ferromagnetic and antiferromagnetic interactions, inequalities analogous to the first Griffiths inequality have been proved with various bond randomness. ${ }^{2-4)}$ In these cases, $J_{B}$ is a random variable, so that it has been proved that the pressure is a monotonic increasing function with respect to some parameter which controls the effect of an interaction term. Similar to the ferromagnetic cases, the inequalities 
may, for example, be used for the proof of the existence of the thermodynamic limit of the pressure density.

As far as we know, however, the inequality analogous to the second Griffiths inequality has only been proved on the Nishimori line, which is a restricted region in the phase diagram of random bond systems. Morita et al first proved the inequalities analogous to both the first and second Griffiths inequalities on the Nishimori line for Ising systems with Gaussian bond randomness. ${ }^{5,6)}$ Using the two inequalities, the existence of the thermodynamic limit for the pressure per unit volume and correlation functions has been proved under various boundary conditions. Relations between the location of multicritical points for various lattices have also been derived. The proof, however, uses not only the gauge theory, but also the properties of the Gaussian distribution, so that the proof cannot be directly applied to the systems with other bond randomness, for example, $\pm J$ bond randomness.

Almost all the rigorous results obtained on the Nishimori line are proved, in essential, only using the properties of the gauge transformation, so that they hold not only for any lattice structure, dimension of the lattice, and range of the interaction, but also for various bond randomness. ${ }^{7,8)}$ For example, related to the second Griffiths inequality, the present author proved that the following inequality holds for finite $\pm J$ Ising systems, $A$ and $B$, on the Nishimori line:

$$
\left[\left\langle S_{0} S_{r}\right\rangle\right]_{A} \geq\left[\left\langle S_{0} S_{r}\right\rangle\right]_{B}
$$

when the system $A$ is obtained from the system $B$ by adding random bonds. ${ }^{9)}$ The above proof was originally carried out for the $\pm J$ Ising models, and used for deriving an inequality about the location of multicritical points of various lattices. However, the same argument can be applied to the systems with other bond randomness, since the proof only uses the gauge theory.

Therefore, it is a natural question whether the existence of the two Griffiths inequalities on the Nishimori line is a special property of Ising systems with Gaussian bond randomness, or they hold without the detail property of the probability distribution of random interactions. The main results of the present paper is that the two Griffiths inequalities do hold on the Nishimori line for Ising systems with various bond randomness which includes both Gaussian and $\pm J$ bond randomness, where the proof essentially uses only the properties of the gauge transformation. Thus, the results obtained from the two inequalities for Ising systems with Gaussian bond randomness ${ }^{4,5}$ do also hold for Ising systems with bond randomness considered in the present paper, especially, for the systems with $\pm J$ bond randomness. 


\section{The Model and the Bond Randomness}

Let us first define several quantities. We treat the Ising spin system described by the Hamiltonian

$$
\mathcal{H}=-\sum_{A \subset V} J_{A} S_{A}
$$

where

$$
S_{A}=\prod_{i \in A} S_{i}
$$

Here $V$ is the set of sites, and the sum over $A$ runs over all subsets of $V$ among which interactions exist. The number of sites in $A$ is arbitrary and may be different from subset to subset. The lattice structure is assumed to be reflected in the choice of $A$ for which $J_{A} \neq 0$. The partition function of this system is written as

$$
Z=\sum_{\left\{S_{i}\right\}} \exp \left(\sum_{A \subset \Omega} \beta_{A} J_{A} S_{A}\right)
$$

where we introduce local inverse temperature, $\beta_{A}$. The reason is that we use local inverse temperature, $\beta_{A}$, as a parameter which controls the effect of the interaction term, $-J_{A} S_{A}$, along the Nishimori line, as will be shown later. To investigate the property of a physical quantity at inverse temperature, $\beta$, we must, of course, set all the local inverse temperature to $\beta_{A}=\beta$. The following argument, however, does not depend on the value of each local inverse temperature, $\beta_{A}$.

Then the pressure of the system is written as

$$
P=[\log Z]
$$

where the configurational average over the distribution of bond randomness is written as $[\cdots]$.

The probability distribution of the random interaction $J_{A}$ is denoted by $P\left(J_{A}\right)$. In this paper, we investigate the case that the probability distribution, $P\left(J_{A}\right)$, satisfies the following two conditions:

$$
P\left(-J_{A}\right)=P\left(J_{A}\right) \exp \left(-2 \beta_{p, A} J_{A}\right)
$$

and

$$
\frac{\partial P\left(J_{A}\right)}{\partial \beta_{p, A}}=\left(J_{A}-\left[J_{A}\right]\right) P\left(J_{A}\right),
$$

where $\beta_{p, A}$ is a parameter which characterizes $P\left(J_{A}\right)$.

Most of the probability distributions of random interactions investigated in the spin glass problems may satisfy the above two conditions. In the case of $\pm J$ distribution with the ferromagnetic bond concentration, $p_{A}\left(p_{A}>1 / 2\right)$, defining $\beta_{p, A}$ as

$$
\exp \left(2 \beta_{p, A} J\right)=\frac{p_{A}}{1-p_{A}}, \quad(J>0)
$$


we may rewrite $P\left(J_{A}\right)$ as

$$
\begin{aligned}
P\left(J_{A}\right) & =p_{A} \delta\left(J_{A}-J\right)+\left(1-p_{A}\right) \delta\left(J_{A}+J\right) \\
& =\frac{\exp \left(\beta_{p, A} J\right)}{2 \cosh \left(\beta_{p, A} J\right)} \delta\left(J_{A}-J\right)+\frac{\exp \left(-\beta_{p, A} J\right)}{2 \cosh \left(\beta_{p, A} J\right)} \delta\left(J_{A}+J\right) \\
& =\frac{1}{2 \cosh \left(\beta_{p, A} J\right)}\left(\delta\left(J_{A}-J\right)+\delta\left(J_{A}+J\right)\right) \exp \left(\beta_{p, A} J_{A}\right) .
\end{aligned}
$$

For the Gaussian distribution with average, $J_{0, A}$, and variance, $\sigma_{A}$, defining $\beta_{p, A}$ as

$$
\beta_{p, A}=\frac{J_{0, A}}{\sigma_{A}^{2}}
$$

we have

$$
\begin{aligned}
P\left(J_{A}\right) & =\frac{1}{\sqrt{2 \pi} \sigma_{A}} \exp \left(\frac{-\left(J_{A}-J_{0, A}\right)^{2}}{2 \sigma_{A}^{2}}\right) \\
& =\frac{1}{\sqrt{2 \pi} \sigma_{A}} \exp \left(\frac{-\sigma_{A}^{2} \beta_{p, A}^{2}}{2}\right) \exp \left(\frac{-J_{A}^{2}}{2 \sigma_{A}^{2}}\right) \exp \left(\beta_{p, A} J_{A}\right)
\end{aligned}
$$

In both cases, we can easily see that $P\left(J_{A}\right)$ satisfies two conditions, eqs. (8) and (9), by direct calculations. It is noted that, in the case of $\pm J$ distribution, $\left[J_{A}\right]=J \tanh \left(\beta_{p, A} J\right)$.

In the present notation, the interaction term, $-J_{A} S_{A}$, satisfies the Nishimori condition when

$$
\beta_{p, A}=\beta_{A}
$$

Namely, when eq. (14) is satisfied for all the subsets $\{A\}$, we may use the properties on the Nishimori line which have originally been proved by Nishimori, using the local gauge transformation. ${ }^{7,8)}$

Here, we show two properties of the probability distribution, $P\left(J_{A}\right)$, which are often used in the rest of the paper. When the function, $f\left(J_{A}\right)$, is an odd function of $J_{A}$, namely, $f\left(-J_{A}\right)=-f\left(J_{A}\right)$, we obtain

$$
\left[f\left(J_{A}\right)\right]=\left[\tanh \left(\beta_{p, A} J_{A}\right) f\left(J_{A}\right)\right] .
$$

Also, when the function, $f\left(J_{A}\right)$, is an even function of $J_{A}$, and monotonic increasing function of $\left|J_{A}\right|$, we have

$$
\left[J_{A} \tanh \left(\beta_{A} J_{A}\right) f\left(J_{A}\right)\right] \geq\left[J_{A} \tanh \left(\beta_{A} J_{A}\right)\right]\left[f\left(J_{A}\right)\right]
$$

The proofs of eq. (15) and ineq. (16) are shown in Appendices A and B, respectively.

\section{The inequalities on the Nishimori line}

Let us clarify the situation. We assume that all the interaction terms satisfy the Nishimori condition, eq. (14). In the following, we investigate the change of the pressure and the correlation functions with respect to arbitrary $\beta_{B}$, and prove the following two inequalities:

$$
\frac{d}{d \beta_{B}}[P] \geq 0
$$


and

$$
\frac{d}{d \beta_{B}}\left[\left\langle S_{C}\right\rangle\right] \geq 0
$$

where we denote the thermal average by angular brackets as $\langle\cdots\rangle$. The above inequalities state that the configurational average of the pressure and the correlation functions are monotonic increasing functions of $\beta_{B}$ on the Nishimori line. Here, we explain the role of $\beta_{B}$. When $\beta_{B}$ is zero, there is no interaction term, $-J_{B} S_{B}$, in the system. Increasing the value of $\beta_{B}$ makes the effect of the interaction term, $-J_{B} S_{B}$, larger, though we consider the restricted case that $\beta_{B}$ always satisfies the Nishimori condition, $\beta_{B}=\beta_{p, B}$. Namely, increasing $\beta_{B}$ for the present system corresponds to increasing the strength of an interaction for ferromagnetic Ising models. Thus, on the Nishimori line, ineqs. (17) and (18), play the same role as the two Griffiths inequalities do for ferromagnetic Ising models.

Next, we briefly explain the procedures of the proof, since the proof of two inequalities can be carried out similarly.

\subsection{The basic transformation of the physical quantity}

First, using the identity,

$$
\exp \left(\beta_{B} J_{B} S_{B}\right)=\cosh \left(\beta_{B} J_{B}\right)\left(1+\tanh \left(\beta_{B} J_{B}\right) S_{B}\right)
$$

we rewrite the physical quantity, $Q$, so that the dependence of $\beta_{B}$ may be seen explicitly. Corresponding to this procedure, we denote the configurational average over the distribution of bond randomness except $J_{B}$ as $[\cdots]^{\prime}$. The thermal average without the Boltzmann factor, $\exp \left(\beta_{B} J_{B} S_{B}\right)$, is also denoted as $\langle\cdots\rangle^{\prime}$.

\subsection{The physical quantity on the Nishimori line}

Next, we rewrite the physical quantity, $[Q]$, on the Nishimori line, using the local gauge transformation. Since we investigate the change of the physical quantity, $[Q]$, in the situation where all the interaction terms including $J_{B}$ always satisfy the Nishimori condition, we may use the properties on the Nishimori line, for example, $\left[\left\langle S_{C}\right\rangle\right]=\left[\left\langle S_{C}\right\rangle^{2}\right]$, in any step of the proof. Actually, we perform the local gauge transformation for all the spin variables, and interactions except $J_{B}$. This can be done, since, in this step, there is no Boltzmann factor, $\exp \left(\beta_{B} J_{B} S_{B}\right)$, and the dependence with respect to $J_{B}$ is explicitly seen, as will be shown later.

\subsection{The change of the physical quantity on the Nishimori line}

Finally, we consider the change of the physical quantity, $[Q]$, with respect to $\beta_{B}$. Using eq. (9), we rewite the total derivative of $[Q]$ by $\beta_{B}$ as

$$
\begin{aligned}
\frac{d}{d \beta_{B}}[Q] & =\frac{d}{d \beta_{B}} \int_{-\infty}^{\infty} d J_{B} P\left(J_{B}\right)[Q]^{\prime} \\
& =\int_{-\infty}^{\infty} d J_{B}\left(\frac{\partial}{\partial \beta_{B}} P\left(J_{B}\right)\right)[Q]^{\prime}+\int_{-\infty}^{\infty} d J_{B} P\left(J_{B}\right)\left[\frac{\partial}{\partial \beta_{B}} Q\right]^{\prime}
\end{aligned}
$$


J. Phys. Soc. Jpn.

Full PAPER

$$
=\left[\left(J_{B}-\left[J_{B}\right]\right) Q\right]+\left[\frac{\partial}{\partial \beta_{B}} Q\right] .
$$

We evaluate the second term of rhs by direct calculations. For the first term of rhs, when $Q$ is an even function of $J_{B}$ and monotonic increasing function of $\left|J_{B}\right|$, we have

$$
\left[\left(J_{B}-\left[J_{B}\right]\right) Q\right]=\left[\left(J_{B} \tanh \left(\beta_{B} J_{B}\right)-\left[J_{B} \tanh \left(\beta_{B} J_{B}\right)\right]\right) Q\right] \geq 0
$$

where we use eq. (15) and ineq. (16). It must be noted that, for the $\pm J$ Ising models, $\mathrm{Q}$ mentioned above becomes constant with respect to $J_{B}$, since $J_{B}^{2}=J^{2}$, so that eq. (21) takes the value, zero.

\section{The proof of the first inequality}

In this section, we prove the first inequality (17) following the procedures shown in the previous section.

\subsection{The basic transformation of the pressure}

Using the identity (19), we rewrite the pressure $[P]$ as

$$
[P]=[\log Z]=\left[\log Z^{\prime}\right]^{\prime}+\left[\log \left(\cosh \left(\beta_{B} J_{B}\right)\right)\right]+\left[\log \left(1+\tanh \left(\beta_{B} J_{B}\right)\left\langle S_{B}\right\rangle^{\prime}\right)\right] .
$$

Here, $Z^{\prime}$ denotes the partition function without the Boltzmann factor, $\exp \left(\beta_{B} J_{B} S_{B}\right)$.

\subsection{The pressure on the Nishimori line}

We expand the third term of rhs of eq.(22) as:

$$
\left[\log \left(1+\tanh \left(\beta_{B} J_{B}\right)\left\langle S_{B}\right\rangle^{\prime}\right)\right]=\sum_{n=1}^{\infty}(-1)^{n-1} \frac{1}{n}\left[\tanh ^{n}\left(\beta_{B} J_{B}\right)\left\langle S_{B}\right\rangle^{\prime} n\right] .
$$

On the Nishimori line, using the local gauge transformation and eq. (15), we can rewrite each odd term of the series as

$$
\left[\tanh ^{2 n-1}\left(\beta_{B} J_{B}\right)\left\langle S_{B}\right\rangle^{\prime(2 n-1)}\right]=\left[\tanh ^{2 n}\left(\beta_{B} J_{B}\right)\left\langle S_{B}\right\rangle^{\prime 2 n}\right]
$$

where, the local gauge transformation is performed for all the spin variables and all the interactions except $J_{B}$. Then, we can further rewrite the third term of rhs of eq.(22) as

$$
\begin{aligned}
{\left[\log \left(1+\tanh \left(\beta_{B} J_{B}\right)\left\langle S_{B}\right\rangle^{\prime}\right)\right] } & =\left[\sum_{n=1}^{\infty} \frac{1}{2 n(2 n-1)} \tanh ^{2 n}\left(\beta_{B} J_{B}\right)\left\langle S_{B}\right\rangle^{\prime 2 n}\right] \\
& =\left[F\left(J_{B}\right)\right],
\end{aligned}
$$

were we define $F\left(J_{B}\right)$ as

$$
\begin{aligned}
F\left(J_{B}\right) & =\frac{1}{2}\left(\left(1+\tanh \left(\beta_{B} J_{B}\right)\left\langle S_{B}\right\rangle^{\prime}\right) \log \left(1+\tanh \left(\beta_{B} J_{B}\right)\left\langle S_{B}\right\rangle^{\prime}\right)\right) \\
& +\frac{1}{2}\left(\left(1-\tanh \left(\beta_{B} J_{B}\right)\left\langle S_{B}\right\rangle^{\prime}\right) \log \left(1-\tanh \left(\beta_{B} J_{B}\right)\left\langle S_{B}\right\rangle^{\prime}\right)\right) .
\end{aligned}
$$

By direct calculation, we have

$$
F\left(-J_{B}\right)=F\left(J_{B}\right)
$$


We also obtain

$$
\frac{\partial}{\partial J_{B}} F\left(J_{B}\right)=\frac{\beta_{B}\left\langle S_{B}\right\rangle^{\prime}}{2 \cosh ^{2}\left(\beta_{B} J_{B}\right)} \log \left(\frac{1+\tanh \left(\beta_{B} J_{B}\right)\left\langle S_{B}\right\rangle^{\prime}}{1-\tanh \left(\beta_{B} J_{B}\right)\left\langle S_{B}\right\rangle^{\prime}}\right) \geq 0, \quad\left(J_{B}>0\right)
$$

regardless of the sign of $\left\langle S_{B}\right\rangle^{\prime}$. Namely, $F\left(J_{B}\right)$ is an even function of $J_{B}$ and monotonic increasing function of $\left|J_{B}\right|$.

\subsection{The change of the pressure on the Nishimori line}

Now, we calculate the total derivative of the pressure, $[P]$, by $\beta_{B}$ on the Nishimori line. First, we have

$$
\frac{d}{d \beta_{B}}[P]=\frac{d}{d \beta_{B}}\left[\log \left(\cosh \left(\beta_{B} J_{B}\right)\right)\right]+\frac{d}{d \beta_{B}}\left[\log \left(1+\tanh \left(\beta_{B} J_{B}\right)\left\langle S_{B}\right\rangle^{\prime}\right)\right] .
$$

The first term of rhs of eq. (29) is directly calculated as

$$
\begin{aligned}
\frac{d}{d \beta_{B}}\left[\log \left(\cosh \left(\beta_{B} J_{B}\right)\right)\right] & =\left[\left(J_{B}-\left[J_{B}\right]\right) \log \left(\cosh \left(\beta_{B} J_{B}\right)\right)\right]+\left[J_{B} \tanh \left(\beta_{B} J_{B}\right)\right] \\
& \geq 0
\end{aligned}
$$

where we use ineq. (21), since $\log \left(\cosh \left(\beta_{B} J_{B}\right)\right)$ is an even function of $J_{B}$, and monotonic increasing function of $\left|J_{B}\right|$.

Similarly, the second term of rhs of eq. (29) is calculated as

$$
\frac{d}{d \beta_{B}}\left[\log \left(1+\tanh \left(\beta_{B} J_{B}\right)\left\langle S_{B}\right\rangle^{\prime}\right)\right]=\left[\left(J_{B}-\left[J_{B}\right]\right) F\left(J_{B}\right)\right]+\left[\frac{\partial}{\partial \beta_{B}} F\left(J_{B}\right)\right] .
$$

Using, ineq. (21), it yields that

$$
\left.\left[\left(J_{B}-\left[J_{B}\right)\right]\right) F\left(J_{B}\right)\right] \geq 0
$$

For the second term of rhs of eq. (31), a direct calculation gives

$$
\left[\frac{\partial}{\partial \beta_{B}} F\left(J_{B}\right)\right]=\left[\frac{J_{B}\left\langle S_{B}\right\rangle^{\prime}}{2 \cosh ^{2}\left(\beta_{B} J_{B}\right)} \log \left(\frac{1+\tanh \left(\beta_{B} J_{B}\right)\left\langle S_{B}\right\rangle^{\prime}}{1-\tanh \left(\beta_{B} J_{B}\right)\left\langle S_{B}\right\rangle^{\prime}}\right)\right] \geq 0,
$$

since it is easily seen that each term in the square brackets of rhs of eq. (33) is nonnegative regardless of the sign of $J_{B}\left\langle S_{B}\right\rangle^{\prime}$. Thus, we obtain the first inequality (17).

\section{The proof of the second inequality}

We can similarly prove the second inequality (18), which states that the correlation function is a monotonic increasing function of $\beta_{B}$.

\subsection{The basic transformation of the correlation function}

Using the identity (19), we rewrite the correlation function as

$$
\left\langle S_{C}\right\rangle=\frac{\left\langle S_{C}\right\rangle^{\prime}+\tanh \left(\beta_{B} J_{B}\right)\left\langle S_{B} S_{C}\right\rangle^{\prime}}{1+\tanh \left(\beta_{B} J_{B}\right)\left\langle S_{B}\right\rangle^{\prime}} .
$$

In this subsection, for simplicity, we define $x_{B}$ and $x_{p . B}$ as

$$
x_{B}=\tanh \left(\beta_{B} J_{B}\right),
$$


and

$$
x_{p, B}=\tanh \left(\beta_{p, B} J_{B}\right) .
$$

We further rewrite the correlation function as

$$
\begin{aligned}
\left\langle S_{C}\right\rangle & =\frac{\left\langle S_{C}\right\rangle^{\prime}+x_{B}\left(\left\langle S_{B} S_{C}\right\rangle^{\prime}-\left\langle S_{B}\right\rangle^{\prime}\left\langle S_{C}\right\rangle^{\prime}\right)-x_{B}^{2}\left\langle S_{B} S_{C}\right\rangle^{\prime}\left\langle S_{B}\right\rangle^{\prime}}{1-x_{B}^{2}\left\langle S_{B}\right\rangle^{\prime 2}} \\
& =\left\langle S_{C}\right\rangle^{\prime}+\frac{x_{B}\left(\left\langle S_{B} S_{C}\right\rangle^{\prime}-\left\langle S_{B}\right\rangle^{\prime}\left\langle S_{C}\right\rangle^{\prime}\right)-x_{B}^{2}\left(\left\langle S_{B} S_{C}\right\rangle^{\prime}-\left\langle S_{B}\right\rangle^{\prime}\left\langle S_{C}\right\rangle^{\prime}\right)\left\langle S_{B}\right\rangle^{\prime}}{1-x_{B}^{2}\left\langle S_{B}\right\rangle^{\prime 2}}
\end{aligned}
$$

Then, the configurational average of the correlation function can be written as

$$
\begin{aligned}
{\left[\left\langle S_{C}\right\rangle\right] } & =\left[\left\langle S_{C}\right\rangle^{\prime}\right]^{\prime}+\left[\frac{\left(\left\langle S_{B} S_{C}\right\rangle^{\prime}-\left\langle S_{B}\right\rangle^{\prime}\left\langle S_{C}\right\rangle^{\prime}\right)\left(x_{B}-x_{B}^{2}\left\langle S_{B}\right\rangle^{\prime}\right)}{1-x_{B}^{2}\left\langle S_{B}\right\rangle^{\prime 2}}\right] \\
& =\left[\left\langle S_{C}\right\rangle^{\prime}\right]^{\prime}+\left[\frac{\left(\left\langle S_{B} S_{C}\right\rangle^{\prime}-\left\langle S_{B}\right\rangle^{\prime}\left\langle S_{C}\right\rangle^{\prime}\right)\left(x_{p, B} x_{B}-x_{B}^{2}\left\langle S_{B}\right\rangle^{\prime}\right)}{1-x_{B}^{2}\left\langle S_{B}\right\rangle^{\prime 2}}\right],
\end{aligned}
$$

where we use eq. (15) for the second term of rhs.

\subsection{The correlation function on the Nishimori line}

On the Nishimori line, since $\beta_{p, B}=\beta_{B}$, and $x_{p, B}=x_{B}$, the correlation function can be rewritten as

$$
\left[\left\langle S_{C}\right\rangle\right]=\left[\left\langle S_{C}\right\rangle^{\prime}\right]^{\prime}+\left[\frac{x_{B}^{2}\left(\left\langle S_{B} S_{C}\right\rangle^{\prime}-\left\langle S_{B}\right\rangle^{\prime}\left\langle S_{C}\right\rangle^{\prime}\right)\left(1-\left\langle S_{B}\right\rangle^{\prime}\right)}{1-x_{B}^{2}\left\langle S_{B}\right\rangle^{\prime 2}}\right] .
$$

Performing the local gauge transformation for all the spin variables and interactions except $J_{B}$, we obtain

$$
\begin{gathered}
{\left[\frac{x_{B}^{2}\left\langle S_{B} S_{C}\right\rangle^{\prime}}{1-x_{B}^{2}\left\langle S_{B}\right\rangle^{\prime 2}}\right]=\left[\frac{x_{B}^{2}\left\langle S_{B} S_{C}\right\rangle^{\prime 2}}{1-x_{B}^{2}\left\langle S_{B}\right\rangle^{\prime 2}}\right],} \\
{\left[\frac{x_{B}^{2}\left\langle S_{B}\right\rangle^{\prime}\left\langle S_{C}\right\rangle^{\prime}}{1-x_{B}^{2}\left\langle S_{B}\right\rangle^{\prime 2}}\right]=\left[\frac{x_{B}^{2}\left\langle S_{B} S_{C}\right\rangle^{\prime}\left\langle S_{B}\right\rangle^{\prime}}{1-x_{B}^{2}\left\langle S_{B}\right\rangle^{\prime 2}}\right]=\left[\frac{x_{B}^{2}\left\langle S_{B} S_{C}\right\rangle^{\prime}\left\langle S_{B}\right\rangle^{\prime}\left\langle S_{C}\right\rangle^{\prime}}{1-x_{B}^{2}\left\langle S_{B}\right\rangle^{\prime 2}}\right],}
\end{gathered}
$$

and

$$
\left[\frac{x_{B}^{2}\left\langle S_{B}\right\rangle^{\prime 2}\left\langle S_{C}\right\rangle^{\prime}}{1-x_{B}^{2}\left\langle S_{B}\right\rangle^{\prime 2}}\right]=\left[\frac{x_{B}^{2}\left\langle S_{B}\right\rangle^{\prime 2}\left\langle S_{C}\right\rangle^{\prime 2}}{1-x_{B}^{2}\left\langle S_{B}\right\rangle^{\prime 2}}\right]
$$

Thus, the second term of rhs of eq. (39) becomes

$$
\left[\frac{x_{B}^{2}\left(\left\langle S_{B} S_{C}\right\rangle^{\prime}-\left\langle S_{B}\right\rangle^{\prime}\left\langle S_{C}\right\rangle^{\prime}\right)\left(1-\left\langle S_{B}\right\rangle^{\prime}\right)}{1-x_{B}^{2}\left\langle S_{B}\right\rangle^{\prime 2}}\right]=\left[\frac{x_{B}^{2}\left(\left\langle S_{B} S_{C}\right\rangle^{\prime}-\left\langle S_{B}\right\rangle^{\prime}\left\langle S_{C}\right\rangle^{\prime}\right)^{2}}{1-x_{B}^{2}\left\langle S_{B}\right\rangle^{\prime 2}}\right] .
$$

Hence, we obtain

$$
\left[\left\langle S_{C}\right\rangle\right]=\left[\left\langle S_{C}\right\rangle^{\prime}\right]^{\prime}+\left[\frac{\tanh ^{2}\left(\beta_{B} J_{B}\right)\left(\left\langle S_{B} S_{C}\right\rangle^{\prime}-\left\langle S_{B}\right\rangle^{\prime}\left\langle S_{C}\right\rangle^{\prime}\right)^{2}}{1-\tanh ^{2}\left(\beta_{B} J_{B}\right)\left\langle S_{B}\right\rangle^{\prime 2}}\right],
$$

where, for the second term of rhs of eq. (44), it is easily seen that the term in the square brackets is an even function of $J_{B}$ and monotonic increasing function of $\left|J_{B}\right|$. 


\subsection{The change of the correlation function on the Nishimori line}

The total derivative of the correlation function by $\beta_{B}$ on the Nishimori line can be written as

$$
\begin{aligned}
\frac{d}{d \beta_{B}}\left[\left\langle S_{C}\right\rangle\right] & =\left[\left(J_{B}-\left[J_{B}\right]\right) \frac{\tanh ^{2}\left(\beta_{B} J_{B}\right)\left(\left\langle S_{B} S_{C}\right\rangle^{\prime}-\left\langle S_{B}\right\rangle^{\prime}\left\langle S_{C}\right\rangle^{\prime}\right)^{2}}{1-\tanh ^{2}\left(\beta_{B} J_{B}\right)\left\langle S_{B}\right\rangle^{\prime 2}}\right] \\
& +\left[\frac{\partial}{\partial \beta_{B}}\left(\frac{\tanh ^{2}\left(\beta_{B} J_{B}\right)\left(\left\langle S_{B} S_{C}\right\rangle^{\prime}-\left\langle S_{B}\right\rangle^{\prime}\left\langle S_{C}\right\rangle^{\prime}\right)^{2}}{1-\tanh ^{2}\left(\beta_{B} J_{B}\right)\left\langle S_{B}\right\rangle^{\prime 2}}\right)\right]
\end{aligned}
$$

Using ineq. (21), the first term of rhs of eq. (45) becomes nonnegative. For the second term of rhs of eq. (45), a direct calculation gives

$$
\begin{aligned}
& {\left[\frac{\partial}{\partial \beta_{B}}\left(\frac{\tanh ^{2}\left(\beta_{B} J_{B}\right)\left(\left\langle S_{B} S_{C}\right\rangle^{\prime}-\left\langle S_{B}\right\rangle^{\prime}\left\langle S_{C}\right\rangle^{\prime}\right)^{2}}{1-\tanh ^{2}\left(\beta_{B} J_{B}\right)\left\langle S_{B}\right\rangle^{\prime 2}}\right)\right] } \\
= & {\left[\frac{2 J_{B} \tanh \left(\beta_{B} J_{B}\right)\left(\left\langle S_{B} S_{C}\right\rangle^{\prime}-\left\langle S_{B}\right\rangle^{\prime}\left\langle S_{C}\right\rangle^{\prime}\right)^{2}}{\cosh ^{2}\left(\beta_{B} J_{B}\right)\left(1-\tanh ^{2}\left(\beta_{B} J_{B}\right)\left\langle S_{B}\right\rangle^{\prime 2}\right)^{2}}\right] \geq 0 }
\end{aligned}
$$

Thus, we obtain the second inequality (18).

\section{Summary and discussion}

We have proved two inequalities, ineqs. (17) and (18), for Ising spin glasses on the Nishimori line with various bond randomness which includes Gaussian and $\pm J$ bond randomness, where the probability distribution of random interactions must satisfy two conditions, eqs. (8) and (9). The two inequalities, which correspond to the Griffiths inequalities for ferromagnetic Ising models, state that, along the Nishimori line, the pressure and the correlation functions are monotonic increasing functions of any $\beta_{B}$ which controls the effect of the interaction term, $-J_{B} S_{B}$. The present results are an generalization of those by Morita et al for Ising systems with Gaussian bond randomness, ${ }^{5}$ ) where the proof uses not only the gauge theory but also the properties of the Gaussian distribution, so that it cannot be directly applied to the systems with other bond randomness. The present proof essentially uses only the gauge theory, so that it holds without the detail property of the probability distribution of random interactions.

Using the present proof, the results obtained from the two inequalities for Ising system with Gaussian bond randomness ${ }^{5,6)}$ can be also derived for the systems with various bond randomness. In the research of the Ising spin glass problems, however, most studies have been carried out for the systems with Gaussian or $\pm J$ bond randomness. Thus, we may insist that the most important physical consequence of the present paper is that it is found that the results obtained for Ising systems with Gaussian bond randomness ${ }^{5,6)}$ do also hold for Ising systems with $\pm J$ bond randomness.

Let us briefly explain several physical consequences on the Nishimori line for regular lattices which can be derived from the two inequalities, though they have already been explained by Morita et al for Ising systems with Gaussian bond randomness. ${ }^{5)}$ 
From the first inequality (17), we can show that the pressure has the well known superadditivity, ${ }^{2,3)}$ namely

$$
[P]_{V} \geq \sum_{i}[P]_{V_{i}}
$$

where $[P]_{V}$ denotes the pressure of the set of sites, $V$, and

$$
V=\sum_{i} V_{i}
$$

since $[P]_{V}$ is obtained from $\sum_{i}[P]_{V_{i}}$ by adding random bonds among $V_{i}$ and $V_{j}$. Thus, we can show the existence of the thermodynamic limit of the pressure density on the Nishimori line under free boundary conditions, assuming invariance by translation with respect to random interactions and the stability boundedness. ${ }^{2.3)}$ For $\pm J$ Ising spin glasses with short range interactions, it is easily seen that the pressure has a definite stable boundedness.

From the second inequality (18), we can show the existence of the thermodynamic limit of the correlation functions on the Nishimori line under free and fixed boundary conditions. For free boundary conditions, when we consider two finite sets of sites, $V$ and $V^{\prime}$ so that $V^{\prime} \supset V$, we get

$$
\left[\left\langle S_{C}\right\rangle\right]_{V^{\prime}} \geq\left[\left\langle S_{C}\right\rangle\right]_{V}
$$

since $V^{\prime}$ is obtained from $V$ by adding random bonds. Thus, we may say that the correlation function is a monotonic increasing function with the system size. With the fact that the correlation function is bounded by unity, we can assert the existence of the thermodynamic limit of the correlation function under free boundary conditions. We can also prove the existence of the thermodynamic limit of the correlation functions under fixed boundary conditions by similar procedure. (See Ref. 5 for details.)

From the second inequality, we can also obtain the relation between the location of the multicritical points. When the lattice $L_{1}$ is obtained from the lattice $L_{2}$ by adding random bonds, we get the following inequality for the magnetization

$$
\left[\left\langle S_{i}\right\rangle\right]_{L_{1}} \geq\left[\left\langle S_{i}\right\rangle\right]_{L_{2}}
$$

from which, we have

$$
T_{\mathrm{c} L_{1}} \geq T_{\mathrm{c}, L_{2}},
$$

where $T_{\mathrm{c}, L_{1}}\left(T_{\mathrm{c}, L_{2}}\right)$ is the temperature of the multicritical point of the lattice, $L_{1}\left(L_{2}\right)$.

Finally, we mention about the relation between ineq. (3) and the second ineq. (18) of the present paper including the work by Morita et al. ${ }^{5)}$ Inequality (3) only states that, for finite systems, the correlation function of some positive $\beta_{B}$ is larger than that of $\beta_{B}=0$, and cannot give the information between the values of the correlation functions of two different positive $\beta_{B}$. Compared to the above fact, from eq. (44), we can explicitly see how the value of the correlation funcion increases as $\beta_{B}$ increases. Thus, ineq. (3) has clearly less information 
than ineq. (18), which comes from the derivative of eq. (44) by $\beta_{B}$. However, ineq. (50), for example, can be derived using only ineq. (3), though it was not explicitly mentioned in Ref. 9. In the proof of ineq. (3), the condition for the probability distribution of random interactions is only one condition, namely, eq. (8), which states that the system has the Nishimori line itself. Thus, following the argument executed in Ref. 5, the existence of the thermodynamic limit of the correlation function on the Nishimori line under free boundary conditions may be proved for all the systems which have the Nishimori line in the phase diagram. The relation between the location of multicritical points of various lattices can also be derived for the same systems.

\section{Acknowledgement}

We thank Satoshi Morita for useful comments.

\section{Appendix A: Derivation of eq. (15)}

In this appendix we explain the derivation of eq. (15). Through Appendices A snd B, we denote the configurational average over the distribution of bond randomness except $J_{A}$ as $[\cdots]^{\prime}$.

Using eq. (8) and the fact that $f\left(J_{A}\right)$ is an odd function of $J_{A}$, we have

$$
\begin{aligned}
{\left[\exp \left(-\beta_{p, A} J_{A}\right) f\left(J_{A}\right)\right] } & =\int_{-\infty}^{\infty} d J_{A} P\left(J_{A}\right) \exp \left(-\beta_{p, A} J_{A}\right)\left[f\left(J_{A}\right)\right]^{\prime} \\
& =\int_{-\infty}^{\infty} d J_{A} P\left(-J_{A}\right) \exp \left(\beta_{p, A} J_{A}\right)\left[f\left(J_{A}\right)\right]^{\prime} \\
& =-\int_{\infty}^{-\infty} d J_{A} P\left(J_{A}\right) \exp \left(-\beta_{p, A} J_{A}\right)\left[f\left(-J_{A}\right)\right]^{\prime} \\
& =-\int_{-\infty}^{\infty} d J_{A} P\left(J_{A}\right) \exp \left(-\beta_{p, A} J_{A}\right)\left[f\left(J_{A}\right)\right]^{\prime} \\
& =-\left[\exp \left(-\beta_{p, A} J_{A}\right) f\left(J_{A}\right)\right],
\end{aligned}
$$

which implies

$$
\left[\exp \left(-\beta_{p, A} J_{A}\right) f\left(J_{A}\right)\right]=0
$$

Thus, we also obtain

$$
\left[\frac{\exp \left(-\beta_{p, A} J_{A}\right)}{\exp \left(\beta_{p, A} J_{A}\right)+\exp \left(-\beta_{p, A} J_{A}\right)} f\left(J_{A}\right)\right]=0,
$$

since $\left(1 /\left(\exp \left(\beta_{p, A} J_{A}\right)+\exp \left(-\beta_{p, A} J_{A}\right)\right) f\left(J_{A}\right)\right)$ is also an odd function of $J_{A}$. Therefore, it yields that

$$
\begin{aligned}
{\left[f\left(J_{A}\right)\right] } & =\left[f\left(J_{A}\right)\right]-2\left[\frac{\exp \left(-\beta_{p, A} J_{A}\right)}{\exp \left(\beta_{p, A} J_{A}\right)+\exp \left(-\beta_{p, A} J_{A}\right)} f\left(J_{A}\right)\right] \\
& =\left[\tanh \left(\beta_{p, A} J_{A}\right) f\left(J_{A}\right)\right]
\end{aligned}
$$


J. Phys. Soc. Jpn.

Full PAPER

\section{Appendix B: Derivation of ineq. (16)}

When two functions, $f\left(J_{A}\right)$ and $J_{A} \tanh \left(\beta_{A} J_{A}\right)$, are both even functions of $J_{A}$ and monotonic increasing functions of $\left|J_{A}\right|$, we have

$$
\left[\left(J_{A} \tanh \left(\beta_{A} J_{A}\right)-J_{0} \tanh \left(\beta_{A} J_{0}\right)\right)\left(f\left(J_{A}\right)-f\left(J_{0}\right)\right)\right] \geq 0,
$$

for any constant value, $J_{0}$, from which, it yields that

$$
\left[J_{A} \tanh \left(\beta_{A} J_{A}\right) f\left(J_{A}\right)\right] \geq J_{0} \tanh \left(\beta_{A} J_{0}\right)\left[f\left(J_{A}\right)\right]+\left(\left[J_{A} \tanh \left(\beta_{A} J_{A}\right)\right]-J_{0} \tanh \left(\beta_{A} J_{0}\right)\right)\left[f\left(J_{0}\right)\right]^{\prime} .
$$

Here, we can choose the value, $J_{0}$, so that it satisfies

$$
\left[J_{A} \tanh \left(\beta_{A} J_{A}\right)\right]=J_{0} \tanh \left(\beta_{A} J_{0}\right) .
$$

Substituting eq. (B.3) into eq.(B.2), we obtain ineq. (16). 


\section{References}

1) R. B. Griffiths: J. Math. Phys. 8 (1967) 478, 484.

2) F. Guerra and F. Toninelli: Commun. Math. Phys. 230 (2002) 71.

3) P. Contucci and S. Graffi: J. Stat. Phys. 115 (2004) 581.

4) P. Contuccci and J. Lebowitz: Ann. Henri Poincare 8 (2007) 1461.

5) S. Morita, H. Nishimori and P. Contucci: J. Phys. A: Math. Gen. 37 (2004) L203.

6) P. Contucci, S. Morita and H. Nishimori: J. Stat. Phys. 122 (2006) 303.

7) H. Nishimori: Prog. Theor. Phys. 66 (1981) 1169.

8) H. Nishimori: Statistical Physics of Spin Glasses and Information Processing: An Introduction, Oxford University Press (Oxford, 2001).

9) H. Kitatani: J. Phys. Soc. Jpn. 63 (1994) 2070. 